\title{
CXCL5 signaling is a shared pathway of neuroinflammation and blood-brain barrier injury contributing to white matter injury in the immature brain
}

Lin-Yu Wang ${ }^{1,2}$, Yi-Fang Tu ${ }^{3,4}$, Yung-Chieh Lin ${ }^{3,4}$ and Chao-Ching Huang ${ }^{3,5,6^{*}}$

\begin{abstract}
Background: In very preterm infants, white matter injury is a prominent brain injury, and hypoxic ischemia (HI) and infection are the two primary pathogenic factors of this injury. Microglia and microvascular endothelial cells closely interact; therefore, a common signaling pathway may cause neuroinflammation and blood-brain barrier (BBB) damage after injury to the immature brain. CXC chemokine ligand 5 (CXCL5) is produced in inflammatory and endothelial cells by various organs in response to insults. CXCL5 levels markedly increased in the amniotic cavity in response to intrauterine infection and preterm birth in clinical studies. The objective of this study is to determine whether CXCL5 signaling is a shared pathway of neuroinflammation and BBB injury that contributes to white matter injury in the immature brain.
\end{abstract}

Methods: Postpartum day 2 (P2) rat pups received lipopolysaccharide (LPS) followed by 90-min HI. Immunohistochemical analyses were performed to determine microglial activation, neutrophil infiltration, BBB damage, and myelin basic protein and glial fibrillary acidic protein expression. Immunofluorescence experiments were performed to determine the cellular distribution of CXCL5. Pharmacological tests were performed to inhibit or enhance CXCL5 activity.

Results: On P2, predominant increases in microglial activation and BBB damage were observed $24 \mathrm{~h}$ after LPS-sensitized $\mathrm{HI}$ induction, and white matter injury (decreased myelination and increased astrogliosis) was observed on P12 compared with controls. Immunohistochemical analyses revealed increased CXCL5 expression in the white matter 6 and $24 \mathrm{~h}$ after insult. Immunofluorescence experiments revealed upregulated CXCL5 expression in the activated microglia and endothelial cells $24 \mathrm{~h}$ after insult. CXCL5 inhibition by SB225002, a selective nonpeptide inhibitor of CXCR2, significantly attenuated microglial activation and BBB damage, increased myelination, and reduced astrogliosis in the white matter after LPS-sensitized HI. In addition, CXCL5-sensitized HI or CXCL5 alone significantly induced BBB damage and white matter injury in association with different neuroinflammation mechanisms. CXCL5-sensitized $\mathrm{HI}$-induced microglial activation and neutrophil infiltration, whereas CXCL5 alone predominately caused neutrophil infiltration.

Conclusions: $C X C L 5$ is a potential biomarker for white matter injury in preterm infants. Pharmacological blockade of CXCL5 signaling that attenuates dysregulated neuroinflammation can be used a therapeutic strategy against white matter injury in the immature brain.

Keywords: CXCL5, CXCR2, Neuroinflammation, Blood-brain barrier, Lipopolysaccharide, Hypoxic ischemia, White matter injury, Immature brain, Preterm infant

\footnotetext{
*Correspondence: huangped@mail.ncku.edu.tw

${ }^{3}$ Department of Pediatrics, National Cheng Kung University Hospital, College

of Medicine, National Cheng Kung University, Tainan 704, Taiwan

${ }^{5}$ Department of Pediatrics, College of Medicine, Taipei Medical University,

Taipei 110, Taiwan

Full list of author information is available at the end of the article
} 


\section{Background}

Cytokines from monocytes and macrophages initiate inflammation by inducing chemokines released from inflammatory and endothelial cells $[1,2]$. Chemokines, the principal factors in leukocyte migration during inflammation, can propagate the process through the recruitment and activation of additional cellular inflammatory mediators, such as macrophages and neutrophils. CXC chemokine ligand 5 (CXCL5) is a small cytokine belonging to the CXC chemokine family that is also known as epithelial neutrophil-activating peptide-78. CXCL5 is expressed in many different cells, including monocytes, endothelial cells, and alveolar epithelial type II cells [3, 4]. It is expressed in various organs, such as the brain, during endotoxemia [5]. CXCL5 is also produced by immune and vascular endothelial cells in response to proinflammatory cytokines through NF-kB activation $[3,6]$.

Cerebral white matter injury is a prominent brain injury and a leading cause of cerebral palsy in preterm infants [7]. The neuropathological hallmark of white matter injury in preterm infants is severe neuroinflammation and focal and diffuse white matter lesions along with astrocytosis and hypomyelination at early and late stages, respectively [7]. During insults, inflammatory cells may exacerbate white matter injury through the production of proinflammatory cytokines [7, 8]. The damaged microvessels may recruit activated leukocytes at the injured white matter through the disrupted blood-brain barrier (BBB), resulting in sustained neuroinflammation, which contributes to the exacerbation of white matter injury through the prolonged production of inflammatory cytokines or chemokines $[9,10]$. Leukocytes and vascular endothelial cells may closely interact; therefore, a common mechanism associated with neuroinflammation and BBB damage and subsequent white matter injury may exist in the immature brain.

CXCL5 levels markedly increased in the amniotic cavity in response to intrauterine infection and preterm birth and in the serum of newborns with hypoxic ischemia (HI) encephalopathy in clinical studies [11, 12]. In addition, elevated CXCL5 levels were observed in the gastric fluids of preterm infants at birth, who subsequently developed severe bronchopulmonary dysplasia [13], and in the surgically resected intestinal samples of preterm infants with necrotizing enterocolitis [14]. However, whether CXCL5 plays a key role in white matter injury of the immature brain remains unknown.

The target cells of damage in white matter injury during the window of vulnerability in preterm infants are O4positive oligodendrocyte progenitors [15]. The predominance of premyelinating oligodendrocytes in postpartum day 2 (P2) rat pups coincides with the high-risk period of white matter injury in very preterm infants [16]. HI and inflammation are the two primary risk factors of white matter injury and cerebral palsy in very preterm infants $[7,10]$. The potentiating effect of infection on HIinduced injury in the white matter of the immature brain has been reported in several studies $[17,18]$. Our previous study on P2 rat pups demonstrated that low-dose lipopolysaccharide (LPS) or 90-min HI alone caused no considerable injury in the cortex or white matter, whereas a combination of the two caused selective white matter injury [18]. These findings suggest that LPS sensitizes HI and selectively causes white matter injury in the immature brain. CXCL5, the chemokine that attracts and activates leukocytes, has been proposed as a potential therapeutic target in several inflammatory diseases [19]. Thus, using pharmacologic approaches in the P2 rat pups (brain maturation status equivalent to human gestation $<28$ weeks), we hypothesized that CXCL5 signaling is the shared pathway associated with neuroinflammation, $\mathrm{BBB}$ damage, and subsequent white matter injury in the immature brain.

\section{Methods}

This study was approved by the Animal Care Committee of National Cheng Kung University. Technicians and investigators blinded to the grouping performed the experiments and quantitative measurements, respectively.

\section{Rat pup model of LPS-sensitized $\mathrm{HI}$ white matter injury}

In this study, 10-12 Sprague-Dawley rat pups per dam were housed with a 12-h light-dark cycle and cared for according to the National Institutes of Health guidelines. The P2 Sprague-Dawley rat pups received intraperitoneal $0.05 \mathrm{mg} / \mathrm{kg}$ LPS (Escherichia coli 0111:B4; SigmaAldrich) or normal saline (NS) injection $3 \mathrm{~h}$ before $\mathrm{HI}$. To avoid LPS-induced body temperature changes, the pups were returned to their dams after LPS or NS administration and housed in an incubator to maintain the body temperature at $33-34{ }^{\circ} \mathrm{C}$ before $\mathrm{HI}$. Subsequently, $\mathrm{HI}$ was induced by ligating the right common carotid artery and inducing hypoxia. The right common carotid artery was permanently ligated under $2.5 \%$ halothane anesthesia. After surgery, the pups were returned to an incubator for 1-h recovery. Subsequently, the rats were placed in airtight 500-mL containers partially submerged in $36{ }^{\circ} \mathrm{C}$ water baths. The containers were humidified with $6.5 \%$ oxygen at a flow rate of $3 \mathrm{~L} / \mathrm{min}$ for $90 \mathrm{~min}$ [18]. Following hypoxia, the pups were returned to their dam and randomly assigned to three groups: control (NS without $\mathrm{HI}, N=12$ ), NS + HI (NS injection $3 \mathrm{~h}$ before HI, $N=12$ ), and LPS + HI (LPS injection $3 \mathrm{~h}$ before HI, $N=12)$.

\section{Pharmacological inhibition of CXCR2 in LPS-sensitized HI} SB225002, a selective nonpeptide inhibitor of CXCR2, the receptor of CXCL5, was used for inhibiting CXCL5 activity [20]. The pups received intraperitoneal injections 
of SB225002 (1 or $3 \mathrm{mg} / \mathrm{kg}$, diluted in NS containing 0.33 \% Tween 80, Cayman Chemical) or vehicle (NS solution containing $0.33 \%$ Tween 80 ) 30 min before LPS administration and immediately after HI. The pups were randomly assigned to four groups: control (pups unexposed to LPS or HI, $N=14$ ), vehicle (NS injections 30 min before LPS administration and immediately after $\mathrm{HI}, N=18$ ), and SB-1 $(1 \mathrm{mg} / \mathrm{kg}, N=14)$ and SB-3 $(3 \mathrm{mg} / \mathrm{kg}, N=18)$ (SB225002 injections 30 min before LPS administration and immediately after HI). The SB225002 dose used in this study was modified from that used by Bento et al. [20].

\section{Intracerebroventricular CXCL5 infusion}

The P2 pups were anesthetized using $2.5 \%$ halothane and intracerebroventricularly infused with recombinant rat CXCL5 $(25 \mu \mathrm{g}$ of CXCL5 dissolved in $50 \mu \mathrm{L}$ of NS, $\mathrm{R} \& \mathrm{D}$ Systems) or NS into the right cerebral hemisphere by using a 30 -gauge needle on a $10-\mu \mathrm{L}$ Hamilton syringe. The location of each injection in relation to the bregma was $2.0 \mathrm{~mm}$ posterior to, $1.5 \mathrm{~mm}$ lateral to, and $2.0 \mathrm{~mm}$ beneath the skull surface [21]. Each pup received $2 \mu \mathrm{L}$ $(1 \mu \mathrm{g})$ or $4 \mu \mathrm{L}(2 \mu \mathrm{g})$ of CXCL5.

Two separate CXCL5 injection-related experiments were performed. In the HI experiment, CXCL5 or the vehicle was infused $3 \mathrm{~h}$ before HI. Accordingly, five groups were formed: control (without LPS or HI, $N=12$ ), LPS + HI (LPS $3 \mathrm{~h}$ before HI, $N=12$ ), NS + HI (NS before HI, $N=12)$, CXCL5 $-1+$ HI $(1-\mu \mathrm{g}$ CXCL5 before HI, $N=12)$, and CXCL5-2 + HI $(2-\mu \mathrm{g}$ CXCL5 before HI, $N=12)$. In the non-HI experiment, two groups were formed: vehicle (NS, $N=12)$ and CXCL5 $(2 \mu \mathrm{g}, N=12)$.

\section{Immunohistochemical analyses}

The pups were sacrificed, and cryo- and paraffin sections were prepared on P3 (6 and $24 \mathrm{~h}$ after insult) and on P12 (10 days after insult), respectively.

The brains were postfixed, dehydrated using graded alcohols, embedded in paraffin, and coronally sectioned (10- $\mu \mathrm{m}$ thick sections) from the genu of the corpus callosum to the end of the dorsal hippocampus. For immunohistochemical staining, four coronal sections, two at the striatum level $(0.26$ and $0.92 \mathrm{~mm}$ posterior to the bregma) and two at the dorsal hippocampus level (3.14 and $4.16 \mathrm{~mm}$ posterior to the bregma), per rat were selected [18] according to the reference planes in a rat brain atlas [22] and assessed for each brain.

For the cell counting (ED1+ microglia and $\mathrm{MPO}(+)$ neutrophils), myelin basic protein (MBP) score and integrated optical density (IOD) measurement (extravascular immunoglobulin G (IgG), glial fibrillary acidic protein (GFAP), and CXC chemokine ligand 5 (CXCL5)) after immunohistochemistry, three visual fields within the medial, middle, and lateral areas in the white matter of hemisphere per section of the four selected sections per brain as described were analyzed (Additional file 1).

Gray matter injury. Nissl-stained sections were scanned and the cross-sectional areas of the striatum, cortex, and hippocampus in the four brain sections described above were assessed manually by tracing the histological area using a computerized image analysis system (Image-Pro 6.0) linked to a Nikon E400 microscope. The total crosssectional area in each brain region (cortex, striatum, and hippocampus) was then calculated in the sections assessed, and the percentage of area loss in the ipsilateral hemisphere versus the contralateral hemisphere was determined for each rat pup $[18,21]$.

White matter injury. MBP staining for assessing myelination and GFAP staining for assessing astrogliosis in the white matter were used as markers of white matter injury. After the permeabilization and blocking of nonspecific binding, sections were first incubated overnight at $4{ }^{\circ} \mathrm{C}$ with the anti-rat MBP monoclonal antibody (1:100; Millipore) or rabbit polyclonal anti-GFAP antibody (1:800; Millipore), rinsed, and incubated for $1 \mathrm{~h}$ at room temperature with the goat anti-rat (1:200; Santa Cruz) or anti-rabbit (1:300; Pierce Biotechnology) biotinylated secondary antibody. Positively stained cells were visualized using the avidin-biotin-peroxidase complex amplification (Pierce Biotechnology) and detected using diaminobenzidine tetrahydrochloride. MBP scores and GFAP signals were analyzed using ImagePro Plus 6.0. Measurements were performed at $\times 100$ and $\times 200$ magnification per visual field $\left(0.579\right.$ and $0.145 \mathrm{~mm}^{2}$, respectively) for MBP scores and GFAP signals, respectively. Three visual fields in the medial, middle, and lateral areas of the white matter in each hemisphere per section and four sections per brain were analyzed and averaged [18, 23].

MBP expression was graded in the three areas within the white matter in each hemisphere per section using a 4-point scoring system, as described previously [18, 23]: 0 , loss of processes and complete loss of capsule; 1 , loss of processes with thinning or breaks in capsule; 2, complete loss of processes with intact capsule; 3, partial loss of processes; and 4, no MBP loss. The scores of each region were summed to obtain a total score (range, 0-12) for each hemisphere. Each section had a total MBP score in the ipsilateral and contralateral hemispheres. The mean IOD values for the GFAP signals in the white matter of the ipsilateral and contralateral hemispheres of each experimental group were compared with those of the control group to obtain the relative IOD ratios.

Ventricular size ratio. The measurement of the ipsilateral ventricle size ratio of the experimental group was modified from our previous study [24]. The ipsilateral ventricle size of area in the four brain sections $(0.26$, $0.92,3.14$, and $4.16 \mathrm{~mm}$ posterior to the bregma [22]) in 
each rat pup were assessed manually by tracing the ipsilateral ventricular area using a computerized image analysis system (Image-Pro 6.0) linked to the E400 microscope. The ipsilateral ventricle size ratio for each experimental rat was respectively calculated from the four brain sections: ipsilateral ventricle area in the experimental group/ the respective ipsilateral ventricle area in the control group. The ipsilateral ventricle size ratios from the four sections were summed up and divided by 4 to obtain the ventricle size ratio for one rat of the experimental group. The averaged ipsilateral ventricle size ratio of the study group were the summed up of the ventricle size ratios from each rat in the same group and divided by the number of rats used in that group (Additional file 1).

\section{Assessment of neuroinflammation and BBB damage}

At 6 and $24 \mathrm{~h}$ after $\mathrm{HI}$, all the animals were perfused before the brains were removed and processed for immunohistochemical staining. The brains were postfixed, dehydrated using $30 \%(w / v)$ sucrose in phosphatebuffered saline (PBS) for 2 days, and coronally sectioned (20 $\mu \mathrm{m}$ thick) from the genu of the corpus callosum to the end of the dorsal hippocampus. Four coronal sections, as described previously, were assessed for each
$\mathbf{A}$

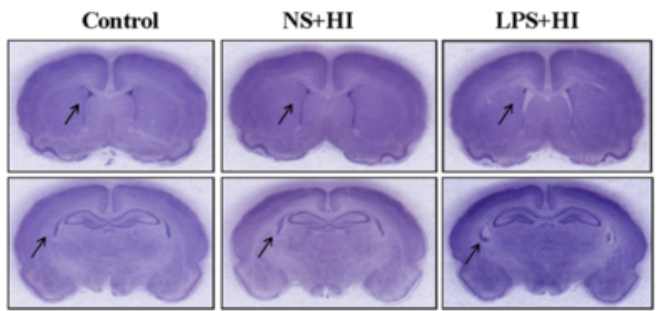

B

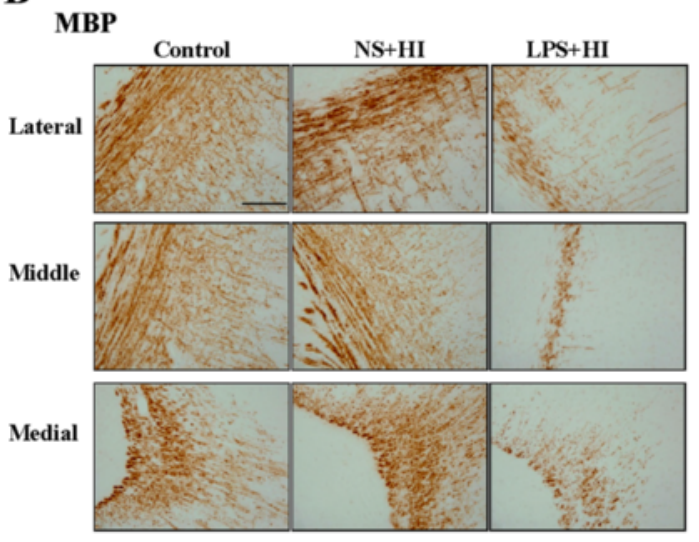

C

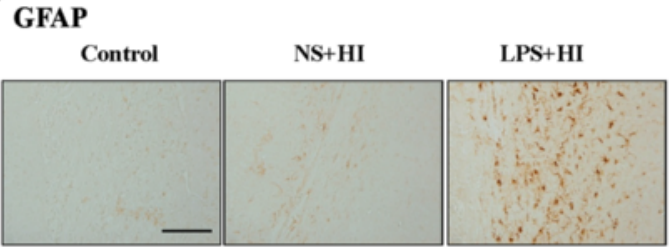

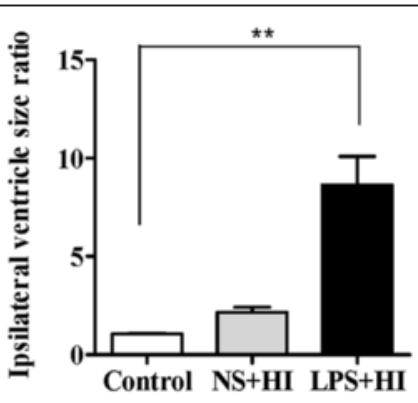
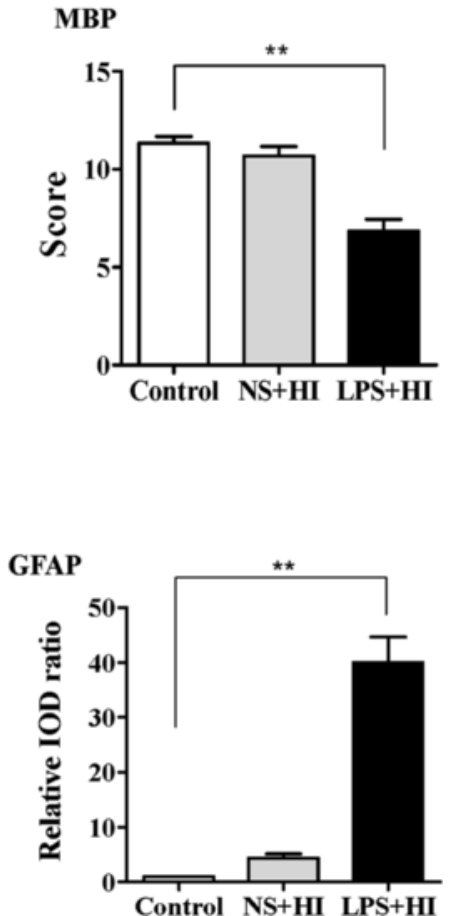

Fig. 1 LPS-sensitized HI caused selective white matter injury. P2 rat pups received LPS ( $0.05 \mathrm{mg} / \mathrm{kg}$ ) or NS 3 h before 90 min of HI. Neuropathological tests were performed on P12 (a, left panel). Nissl staining revealed no significant injury in the gray matter of the control $(n=6)$, NS $+\mathrm{HI}(n=6)$, and LPS $+\mathrm{HI}(n=6)$ groups. Upper panel: brain section at the level of striatum $(0.26 \mathrm{~mm}$ posterior to the bregma) and lower panel: the section at the level of dorsal hippocampus (3.14 mm posterior to the bregma). The LPS + HI group had significantly higher ipsilateral ventricular size ratios (a, right panel), lower myelination (MBP) (b), and higher astrogliosis (GFAP) (c) in the white matter than those of the control group. Scale bar $=100 \mu \mathrm{m}$ for MBP and GFAP. Values are means \pm SEMs. ${ }^{*} p<0.01$. Values are means \pm SEMs. NS, normal saline; LPS, lipopolysaccharide; HI, hypoxic ischemia; MBP, myelin basic protein; GFAP, glial fibrillary acidic protein 
brain. Immunoglobulin G (IgG) extravasation was used as an indicator of BBB permeability [18, 23, 25, 26], and MPO immunoreactive neutrophils were used for detecting neutrophil infiltration. Specific primary antibodies used were the rabbit anti-rat CXCL5 antibody (1:100; R\&D Systems), mouse anti-rat ED1 antibody (1:100, Millipore), rabbit anti-rat MPO antibody (1:100; Abcam), and horseradish peroxidase-conjugated goat anti-rat IgG (1:200; Millipore). Biotinylated secondary antibodies were anti-rabbit and anti-mouse IgGs (all 1:200; Pierce Biotechnology). Biotin-peroxidase signals were detected

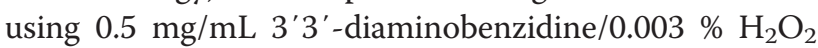
(Dako, Carpinteria) as a substrate. Results were recorded using a microscope (BX51; Olympus).

Quantification was performed at $\times 400$ magnification per visual field $\left(0.0356 \mathrm{~mm}^{2}\right)$ for CXCL5 signals and ED1 $(+)$ microglia and at $\times 200$ magnification per visual field $\left(0.145 \mathrm{~mm}^{2}\right)$ for the $\mathrm{MPO}(+)$ neutrophils and extravascular IgG signals. Three visual fields within the medial, middle, and lateral areas in the white matter of each hemisphere per section and four sections per brain were analyzed and averaged. The mean IOD values in the white matter of the ipsilateral and contralateral hemispheres of each experimental group were compared with those of the control group to obtain the relative IOD ratios.

\section{Fluorescence immunocytochemical analyses}

After blocking $(\times 1 \mathrm{PBS}, 2 \%$ normal goat serum, and $0.1 \%$ Triton X-100) for $1 \mathrm{~h}$, the sections were incubated overnight at $4{ }^{\circ} \mathrm{C}$ with a mixture of any two of the following primary antibodies: anti-CXCL5 (1:50; abcam), anti-ED1 (1:100; Chemicon, Temecula, CA), anti-O4 IgM (1:100; Chemicon), anti-GFAP (1:200; Chemicon), antineuronal nuclear antigen (NeuN) (1:200; Millipore Bioscience Research Reagents), and anti-RECA (1:100; abcam). The sections were washed three times with $0.1 \mathrm{M}$ PBS and incubated with Alexa Fluor 594 anti-mouse IgG/ IgM or Alexa Fluor 488 anti-rabbit IgG (1:400; Invitrogen) for $1 \mathrm{~h}$ at room temperature. The fluorescence signals were detected, and the results were recorded using a microscope (E400; Nikon Instech) at excitation-emission wavelengths of 596-615 nm (Alexa Fluor 594, red) and 470-505 nm (Alexa Fluor 488, green) [18, 23].

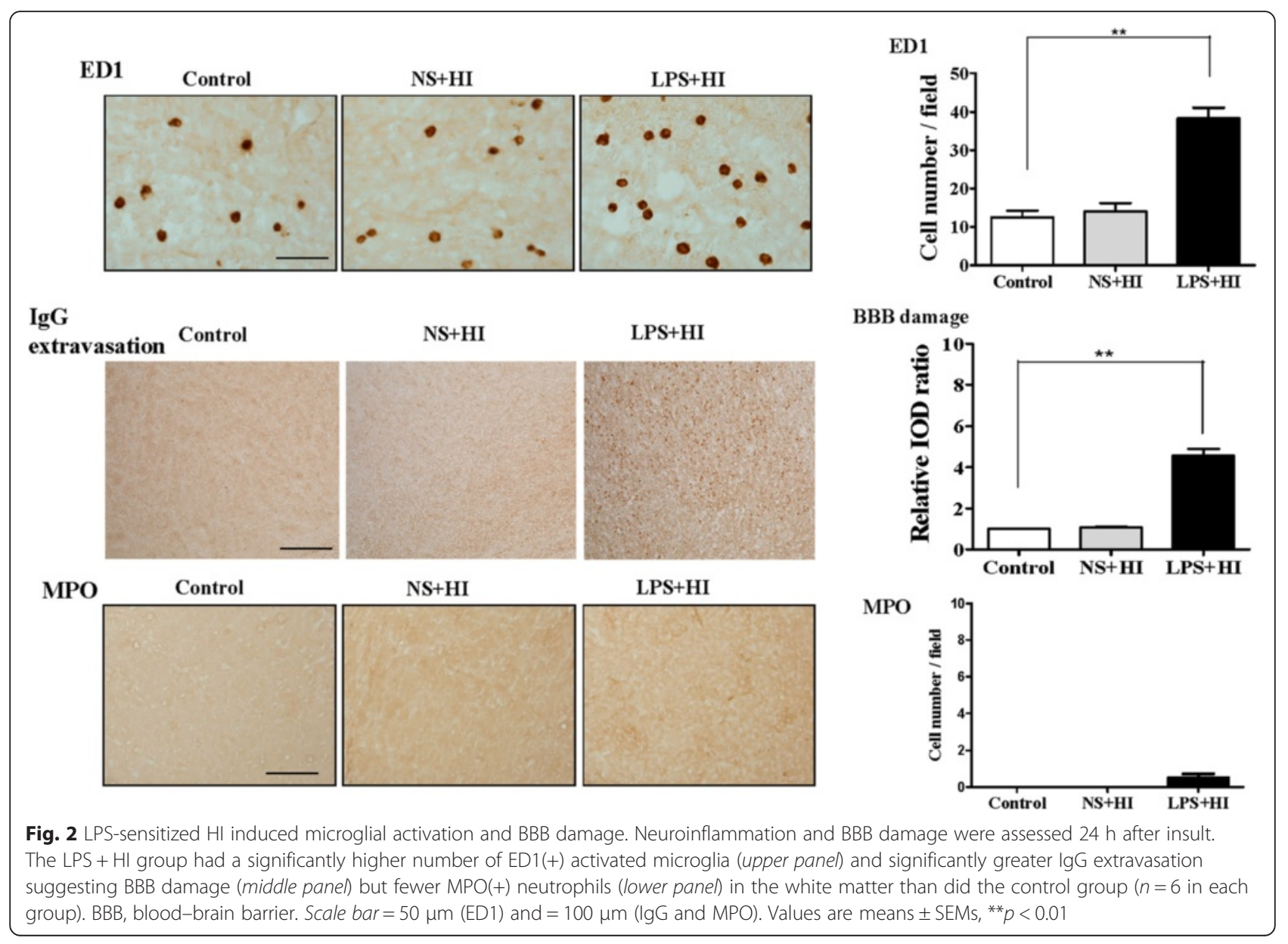




\section{Western blot analysis}

The rat pups were sacrificed 3, 6, and $24 \mathrm{~h}$ after insult for Western blot analysis. Ipsilateral cerebral white matter tissues were homogenized in a cold lysis buffer, and the protein concentrations were determined using a Bio-Rad Protein Assay kit (Bio-Rad Laboratories). Samples were separated using 18 \% SDS-PAGE and blotted electrophoretically onto polyvinylidene fluoride membranes. The membranes were incubated with primary antibodies: antirat CXCL5 (1:400; R\&D Systems) or anti-actin (1:1000; Chemicon). Immunoreactivity was detected using the horseradish peroxidase-conjugated anti-mouse IgG antibody (1:10,000; Calbiochem) and visualized using enhanced chemiluminescence (Pierce Biotechnology). VisionWorks LS analysis software (Ultra-Violet Products Ltd) was used for densitometry analysis, and CXCL5 levels were assessed after they were normalized to actin expression [21].

\section{Statistical analysis}

Statistical significance $(p<0.05)$ was determined using the Kruskal-Wallis test, and Dunn's method was used for post hoc comparisons. Continuous data were presented as means \pm standard errors of the mean (SEMs).

\section{Results}

LPS-sensitized HI caused microglial activation, BBB damage, and white matter injury

We first established the sensitization effect of LPS on $\mathrm{HI}$-induced white matter injury in the P2 rat pups. On P12, Nissl staining revealed no significant neuronal damage in the cortex, striatum, and hippocampus in the NS $+\mathrm{HI}$ and LPS + HI groups (Fig. 1a, left panel). By contrast, the LPS + HI group, but not the NS + HI group, had significantly higher ipsilateral ventricular size ratios (Fig. 1a, right panel), lower myelination (MBP staining, Fig. 1b), and higher astrogliosis (GFAP staining, Fig. 1c) in the white matter of the ipsilateral cerebral hemisphere than those of the control group. Resting microglia were identified as ramified microglia with long processes, whereas primed or activated microglia were identified as microglia that were more rounded, with retracted and shorter processes. At $24 \mathrm{~h}$ after insult, the LPS $+\mathrm{HI}$, but

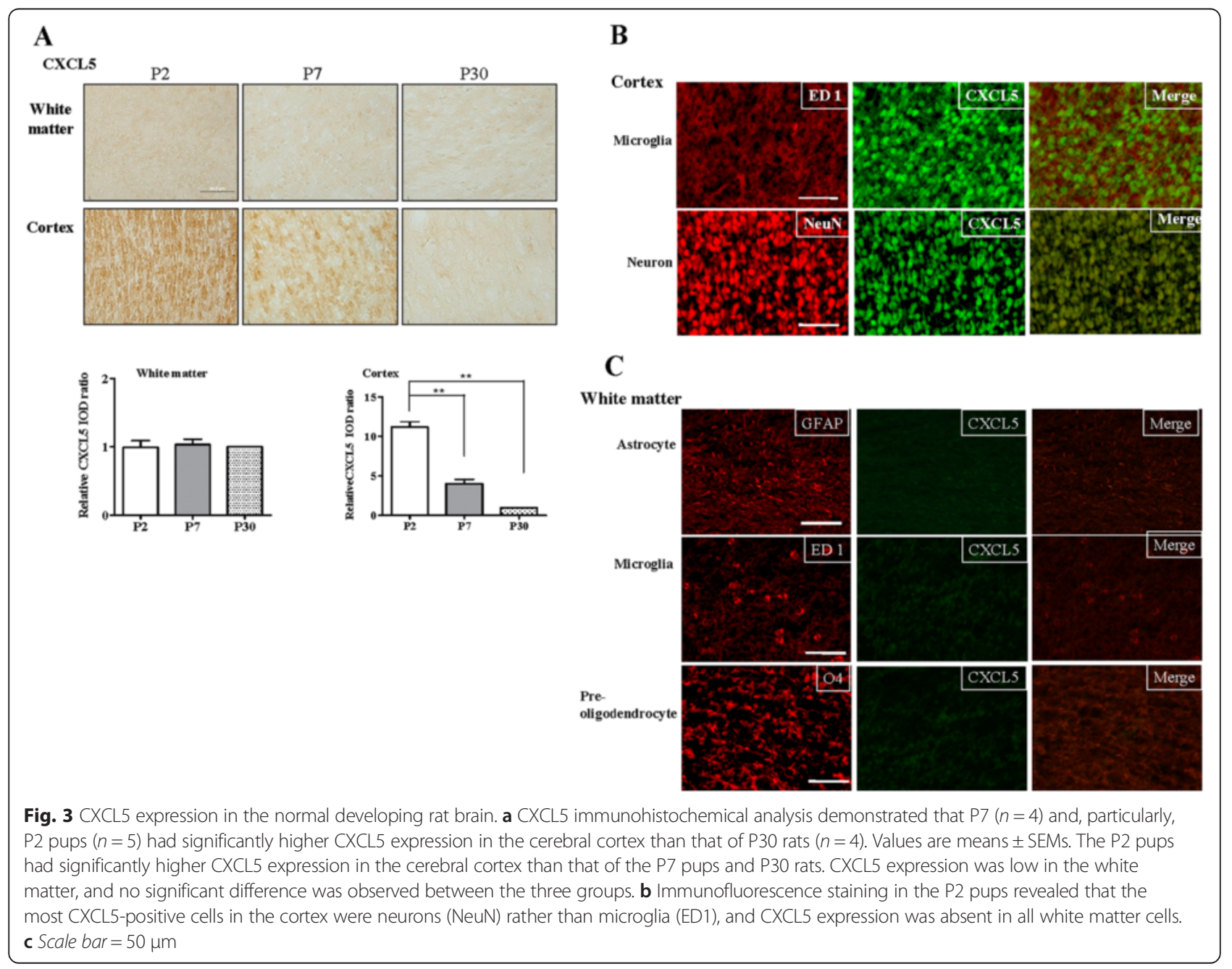


not the NS + HI group, had a significantly higher number of $\mathrm{ED} 1(+)$ activated microglia (Fig. 2, upper panel) and significantly increased BBB damage (Fig. 2, middle panel) but fewer $\mathrm{MPO}(+)$ neutrophils (Fig. 2, lower panel) in the white matter compared with the control group.

\section{LPS-sensitized HI upregulated CXCL5 expression in the} microglia and vascular endothelial cells in the white matter

In the normal developing brain of the P7 and, particularly, P2 pups, significantly higher CXCL5 expression was observed in the cerebral cortex compared with that of the P30 rats (Fig. 3a). By contrast, the CXCL5 expression was low in the white matter, and no significant difference was observed between the P2, P7, and P30 rats.
Immunofluorescence of the P2 cortex revealed that most of the $\mathrm{CXCL}^{+}$cells were $\mathrm{NeuN}(+)$ neurons and not microglia (Fig. 3b). By contrast, CXCL5 was not expressed in any white matter cells (Fig. 3c).

Western blots of the white matter revealed that the LPS + HI group had significantly higher CXCL5 expression at 6 and $24 \mathrm{~h}$ after $\mathrm{HI}$ than that of the controls (Fig. 4a). Immunohistochemical analyses confirmed that the LPS + HI group had significantly higher CXCL5 immunoreactivity in the white matter at $6 \mathrm{~h}$ and, particularly, $24 \mathrm{~h}$ after $\mathrm{HI}$ than that of the control group (Fig. 4b). Immunofluorescence of the white matter $24 \mathrm{~h}$ after LPS-sensitized HI revealed that CXCL5 was upregulated mainly in the ED1(+) microglia and RECA(+) vascular endothelial cells and not in astrocytes or O4(+) preoligodendrocytes (Fig. 4C).

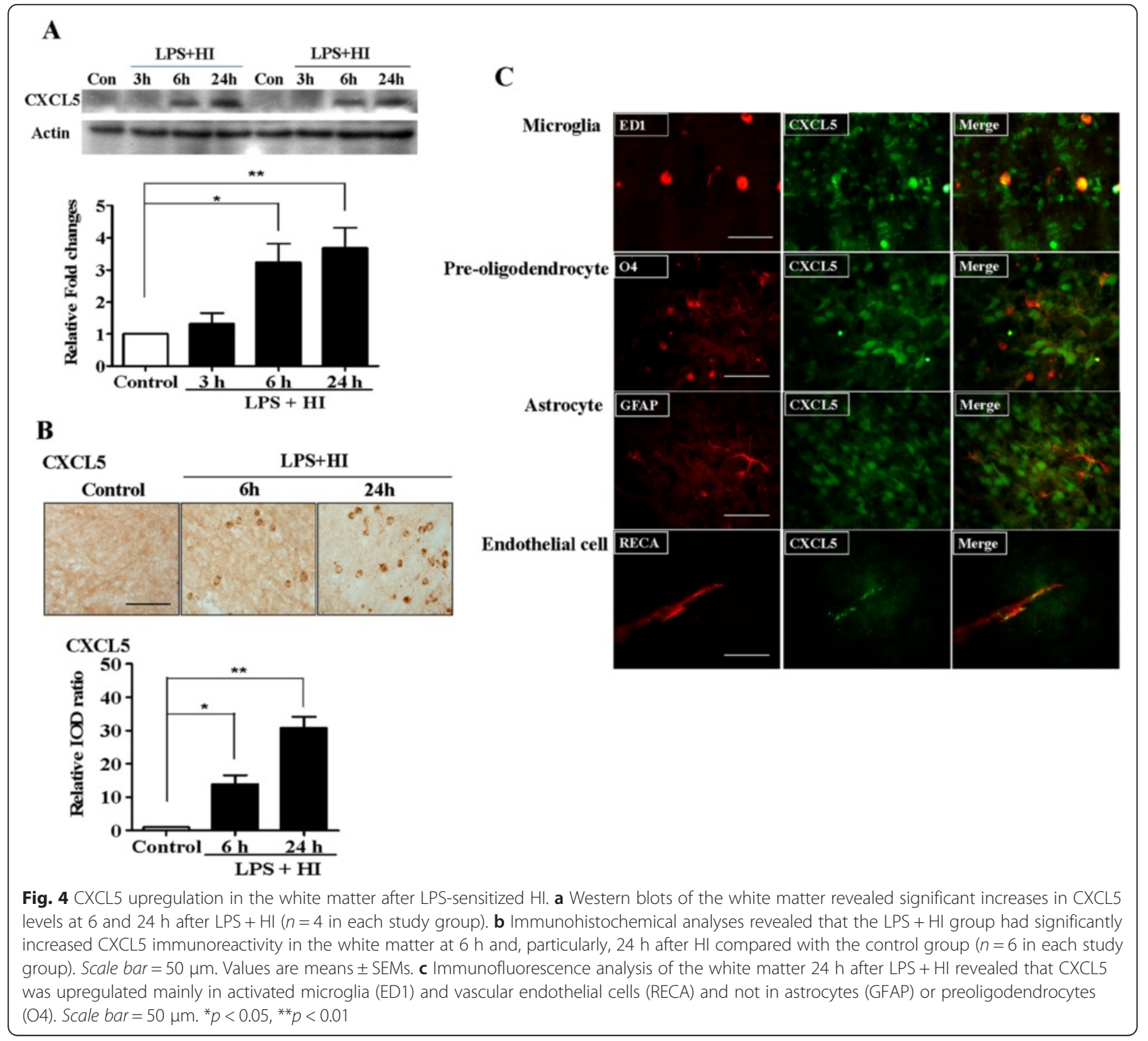



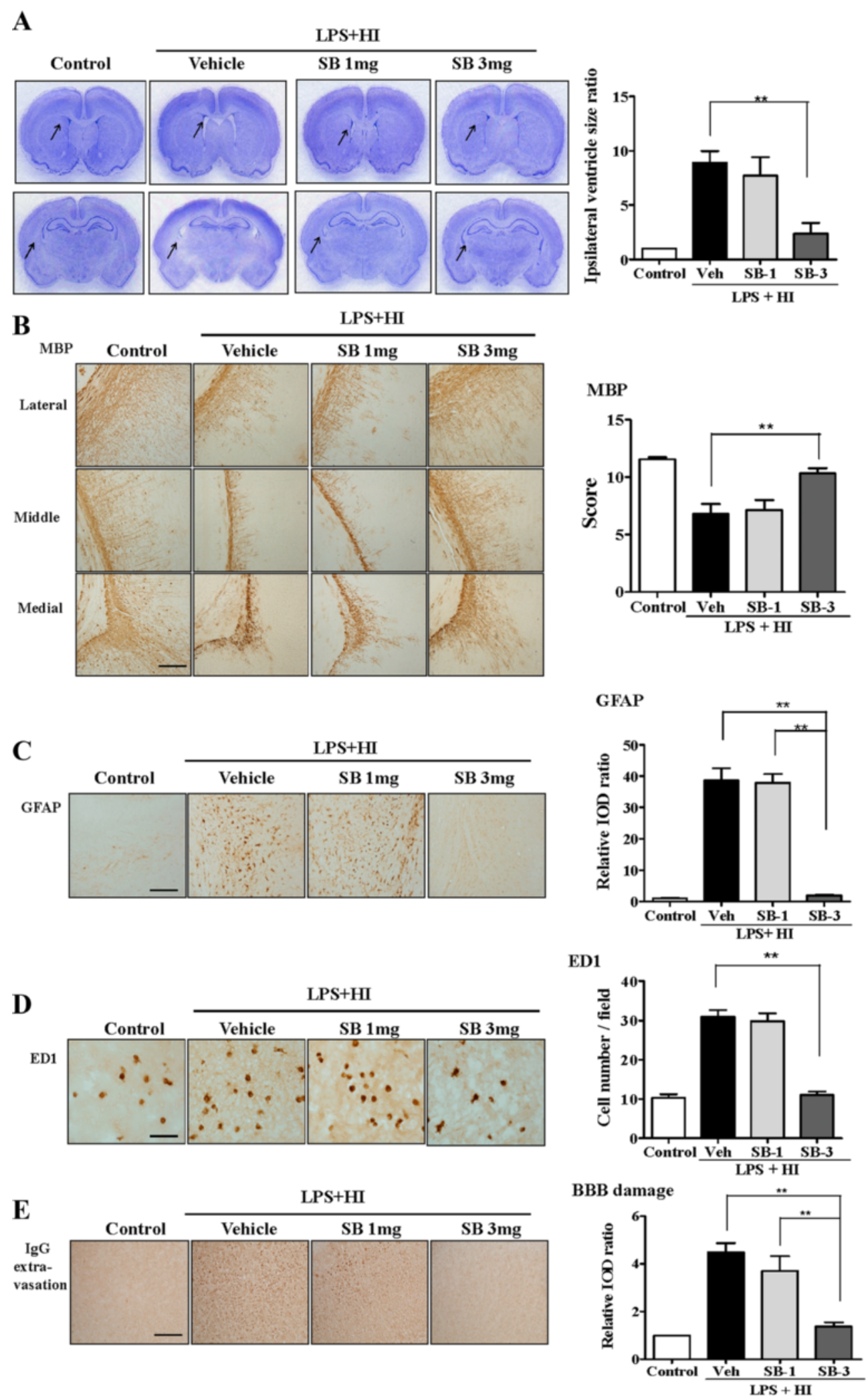

Fig. 5 (See legend on next page.) 
(See figure on previous page.)

Fig. 5 Pharmacological inhibition of CXCR2 significantly attenuated microglial activation and BBB damage and protected against white matter injury after LPS-sensitized HI. A selective nonpeptide inhibitor of CXCR2, SB22 5002, was used to examine the role of the CXCL5-CXCR2 pathway in white matter injury after LPS-sensitized HI. After LPS + HI on P2, the SB-3 group $(n=9)$, but not the SB-1 group $(n=7)$, had significantly reduced ipsilateral ventricular size ratios (a), increased myelination (MBP) (b), and reduced astrogliosis (GFAP) (c) in the white matter on P12 compared with the vehicle-treated pups $(n=9)$. Scale bar $=100 \mu \mathrm{m}$. The SB-3 group $(n=9)$, but not the SB-1 group $(n=7)$, had a significantly reduced number of activated microglia (ED1) (d) and significantly lower BBB damage (IgG extravasation) (e) in the white matter compared with the vehicle group $(n=9)$ at $24 \mathrm{~h}$ after HI. Scale bar $=50 \mu \mathrm{m}(\mathrm{ED} 1)$ and $100 \mu \mathrm{m}(\mathrm{lgG})$; values are means \pm SEMs, ${ }^{* *} p<0.01$

CXCR2 inhibition attenuated microglial activation, reduced $B B B$ damage, and protected against white matter injury after LPS-sensitized $\mathrm{HI}$

We used SB225002, a selective nonpeptide inhibitor of CXCR2, for examining the role of the CXCL5-CXCR2 pathway in white matter injury after LPS-sensitized HI. On P12, the SB-3 group, but not the SB-1 group, had significantly lower ipsilateral ventricular size ratios (Fig. 5a), higher MBP scores (Fig. 5b), and lower astrogliosis (Fig. 5c) in the white matter than those of the vehicle-treated group. The SB-3 group, but not the SB-1 group, had a significantly lower number of ED1(+) activated microglia (Fig. 5d) and significantly reduced $\mathrm{BBB}$ damage (Fig. 5e) in the white matter $24 \mathrm{~h}$ after insult compared with the vehicle group. These results suggest that the CXCL5-CXCR2 pathway plays an important role in microglial activation, BBB damage, and subsequent white matter injury after LPS-sensitized HI in the immature brain.

\section{CXCL5-sensitized $\mathrm{HI}$ caused microglial and neutrophil infiltration, BBB damage, and white matter injury}

Subsequently, we examined whether CXCL5-sensitized HI caused white matter injury in P2 pups. On P12, the $2-\mu \mathrm{g}$ CXCL5 + HI group, but not the 1- $\mu$ g CXCL5 + HI group, had significantly higher ipsilateral ventricle size ratios (Fig. 6a), lower MBP expression (Fig. 6b), and higher astrogliosis (Fig. 6c) in the white matter area compared with the NS + HI or control group. Twentyfour hours after HI, the 2- $\mu$ g CXCL5 + HI and LPS + HI groups had a significantly higher number of ED1(+) microglia (Fig. 6d) and significantly greater BBB damage (Fig. 6e) in the white matter compared with the NS + HI and control groups. By contrast, the $2-\mu \mathrm{g}$ CXCL5 + HI group, but not the LPS + HI group, had significantly higher $\mathrm{MPO}(+)$ neutrophil infiltration (Fig. 6d) in the white matter than that of the NS $+\mathrm{HI}$ and control groups. These results suggested that along with HI, CXCL5 induced microglial activation and neutrophil infiltration, increased BBB damage, and caused white matter injury.

\section{CXCL5 alone increased neutrophil infiltration and BBB damage and caused white matter injury}

We examined whether CXCL5 alone induced white matter injury in the P2 pups. On P12, the CXCL5 group had significantly higher ipsilateral ventricle size ratios (Fig. 7a), lower MBP expression (Fig. 7b), and higher astrogliosis (Fig. 7c) in the white matter than those of the vehicle group. At $24 \mathrm{~h}$ postinjection, no detectable ED1(+) microglia (Fig. 7d, upper panel) were observed in the white matter of the NS and CXCL5 groups. By contrast, the CXCL5 group had a significantly higher number of $\mathrm{MPO}(+)$ neutrophils (Fig. 7d, middle panel) and greater BBB damage (Fig. 7d, lower panel) compared with the vehicle group. These findings suggested that CXCL5 alone was sufficient to induce significant neutrophil accumulation and BBB damage and cause white matter injury.

\section{Discussion}

$\mathrm{HI}$ and inflammation are the two major pathogenetic mechanisms of white matter injury in very preterm infants [7, 17, 27]. Our previous study [18] on P2 rat pups had demonstrated that low-dose LPS or 90-min HI alone caused no significant white matter injury. The LPStreated or 90-min HI pups also showed no evidence of microglial activation or $\mathrm{BBB}$ breakdown in the white matter. In the present study, LPS-sensitized HI induced microglial activation and BBB damage in the P2 rat pups and caused long-term white matter injury (increased ipsilateral ventricular size and astrogliosis and decreased myelination). After LPS-sensitized HI, the CXCL5 levels were markedly upregulated in the white matter and expressed mainly in the microglia and vascular endothelial cells. Attenuating CXCL5 function by inhibiting CXCR2 significantly attenuated microglial activation, reduced BBB damage, and protected against white matter injury. Furthermore, CXCL5 administration with HI or CXCL5 alone caused significant BBB damage and white matter injury in association with different neuroinflammatory mechanisms. CXCL5 with $\mathrm{HI}$ induced microglial activation and neutrophil infiltration, whereas CXCL5 alone caused predominant neutrophil infiltration. These results suggest that CXCL5-mediated signaling pathway plays an essential role in neuroinflammatory activation, BBB disruption, and subsequent white matter injury in the immature brain.

Neuroinflammation and BBB disruption have been associated with the severity of HI injury in the immature brain [7-9, 18, 23, 28, 29]. Neuroinflammation is the neuropathological hallmark of white matter injury in 

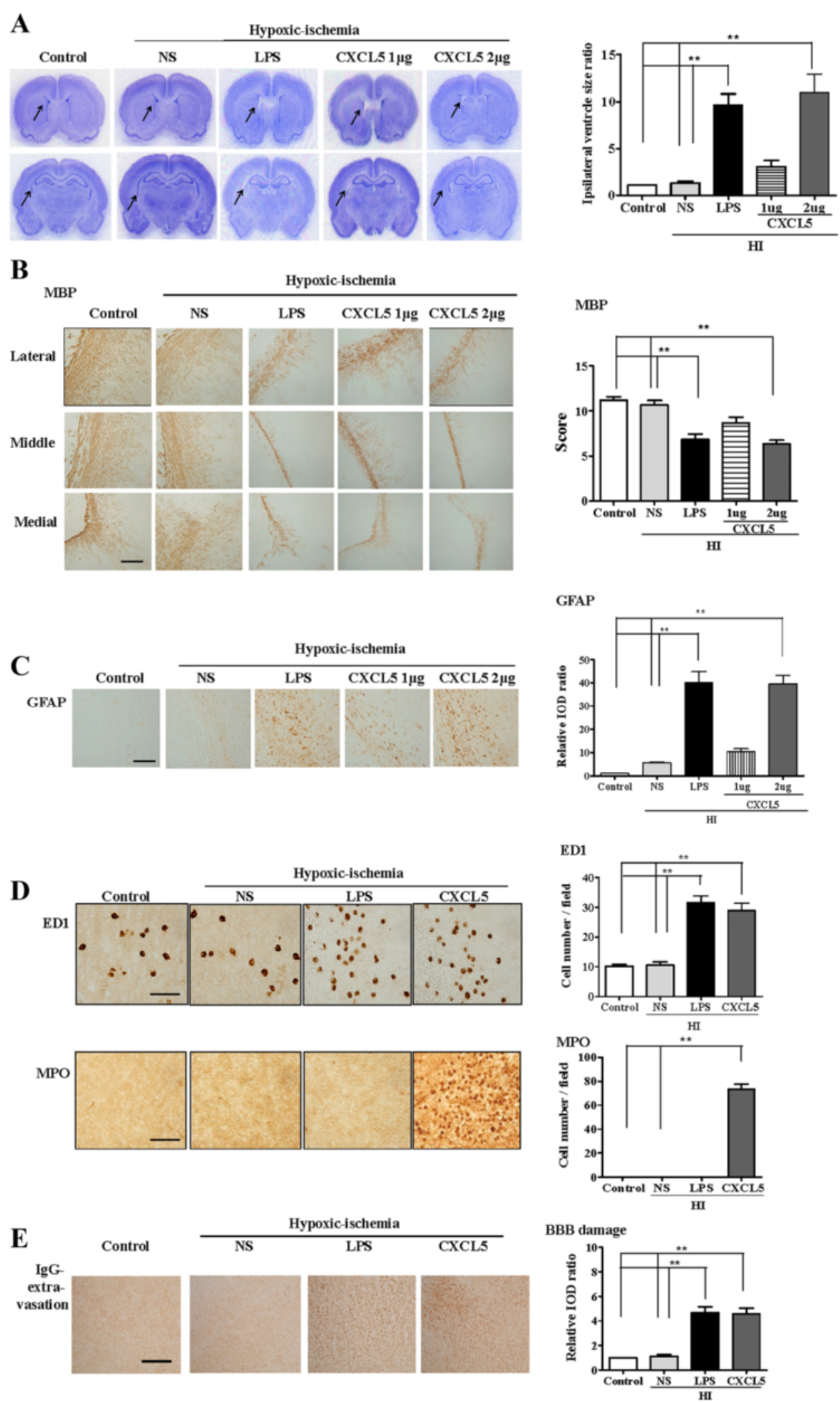

Fig. 6 (See legend on next page.) 


\section{(See figure on previous page.)}

Fig. 6 CXCL5-sensitized HI induced microglial activation, neutrophil infiltration, and BBB damage and caused white matter injury. Intracerebroventricular infusion of recombinant CXCL5 (1 or $2 \mu \mathrm{g}$ ) or NS followed by HI on P2 showed that the $2-\mu \mathrm{g} C X C L 5+H$ group $(n=6)$, but not the $1-\mu \mathrm{g} C X C L 5+H \mathrm{H}$ group $(n=6)$, had significantly higher ipsilateral ventricle size ratios (a), lower myelination (MBP) (b), and higher astrogliosis (GFAP) (c) in the white matter compared with the NS $(n=6)$ and the control groups $(n=6)$ on P12. Scale bar $=100 \mu \mathrm{m}$. At $24 \mathrm{~h}$ after HI, the 2- $\mu \mathrm{g} C X C L 5+H I(n=6)$ and LPS $+\mathrm{HI}$ groups $(n=6)$ had a significantly higher number of activated microglia (ED1) (d) and BBB damage (e) in the white matter compared with the NS ( $n=6)$ and control groups $(n=6)$. The $2-\mu \mathrm{g}$ CXCL5 + HI group, but not the LPS + Hl group, had significantly increased neutrophil infiltrations (MPO) (d) in the white matter. Scale bar $=50 \mu \mathrm{m}$ (ED1) and $100 \mu \mathrm{m}$ (IgG and MPO); values are means \pm SEMs, ${ }^{* *} p<0.01$

preterm infants [7, 29] and may exacerbate injury through BBB damage $[9,30]$. Inflammation involves microglial and endothelial cell activation, leading to proinflammatory cytokine and endothelial adhesion molecule secretion, and is associated with vascular tight junction modification [30]. The damaged microvessels may recruit activated leukocytes at the injured site of the brain through the disrupted $\mathrm{BBB}$, leading to sustained neuroinflammation, which in turn further damages the brain $[9,29,30]$. Neuroinflammation and BBB damage may be two mutually potentiating mechanisms for white matter injury in the developing brain. Our study demonstrated for the first time that LPSsensitized HI-induced CXCL5 expression, CXCL5 in association with $\mathrm{HI}$, and CXCL5 alone play essential roles in neuroinflammation, BBB damage, and subsequent white matter injury in the immature rat brain.

Chemokines rapidly secreted within the central nervous system in response to injuries and infections contribute to host defense mechanisms and disease progression. CXCL5 released from endothelial cells [31] and inflammatory monocytes [4] can propagate the proinflammatory process through the recruitment and activation of additional cellular mediators of inflammation, such as macrophages and neutrophils. Aberrant CXCL5 levels have been detected in various acute and chronic diseases, such as microbial infections, rheumatoid arthritis, obesity, and inflammatory bowel diseases $[3,4,32]$. Elevated CXCL5 expression was observed in many complications of preterm infants $[11,13,14]$. High CXCL5 levels were observed in clinical chorioamnionitis of preterm births [11] and in surgically resected tissue samples of necrotizing enterocolitis from preterm infants [14]. In addition, preterm infants with moderate or severe bronchopulmonary dysplasia had significantly higher CXCL5 levels in the gastric fluid than those with no or mild bronchopulmonary dysplasia did [13]. Currently, information regarding the role of CXCL5 in white matter injury among preterm infants is scant. Our experimental study provides the first evidence that CXCL5 is markedly upregulated and causes white matter injury after LPS-sensitized HI in P2 immature rat brains. In addition, CXCL5, in association with $\mathrm{HI}$ or alone, causes marked white matter injury. These findings suggest that CXCL5 may play a critical role in the pathogenesis of white matter injury of the immature brain. Additional clinical studies are warranted to test whether CXCL5 is an essential biomarker predicting the occurrence of white matter injury and its long-term outcome in preterm infants.

Blocking CXCL5 activity using the CXCR2 antagonist SB225002 is highly effective in ameliorating acute experimental colitis [20] and ischemic brain injury [33]. CXCR2 is a chemokine receptor expressed on endothelial cells, oligodendrocytes, and various immune cells [34] and is essential for neutrophil recruitment at tissue injury- or infection-induced inflammation sites. CXCR2 and its ligands have been implicated in several inflammation-mediated diseases. CXCR2 has been associated with inflammation and myelin disorders [2]. Antagonism of the chemokine receptor CXCR2 has been proposed as a therapeutic strategy for inflammatory diseases [34]. Furthermore, the role of CXCR2 in myeloid and nonmyeloid cells in association with the destruction and repair of myelin has been demonstrated in an animal model of demyelination [35]. Myelin repair was accelerated by inactivating CXCR2 on nonhematopoietic cells [35]. In our experimental study, CXCL5 levels were markedly upregulated, particularly in the microglia and vascular endothelial cells of the white matter of the immature brain after LPS-sensitized HI. CXCL5 upregulation in the microglia and vascular endothelial cells may contribute to neuroinflammation and $\mathrm{BBB}$ damage and subsequent white matter injury, potentially through CXCR2, because attenuating CXCL5 activity by inhibiting CXCR2 significantly attenuated neuroinflammation and BBB damage and protected against white matter injury in the immature brain. Furthermore, after LPSsensitized HI, CXCL5 is a potent chemoattractant for microglia, which may enhance neuroinflammation by binding and signaling through its receptor CXCR2 and further disrupting the BBB integrity. These findings suggest that CXCL5-CXR2 signaling is not only the shared mechanism of neuroinflammation and BBB disruption but also a promising drug target for clinical white matter injury in the immature brain.

In the mitogen-activated protein kinase family, c-Jun $\mathrm{N}$-terminal kinases (JNKs) are crucial stress-responsive kinases that control essential processes, such as inflammation and apoptosis. JNK signaling is triggered by extracellular signals, such as cytokines and environmental stresses 
A

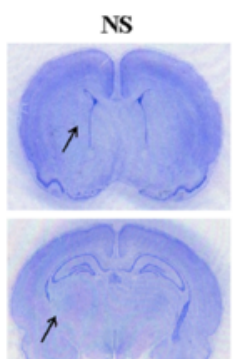

B

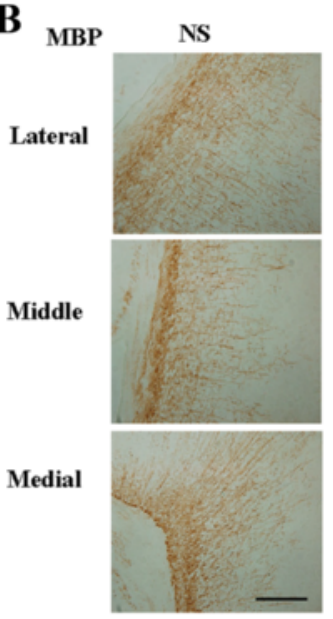

C

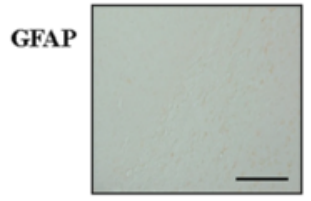

D

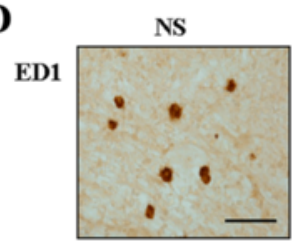

MPO
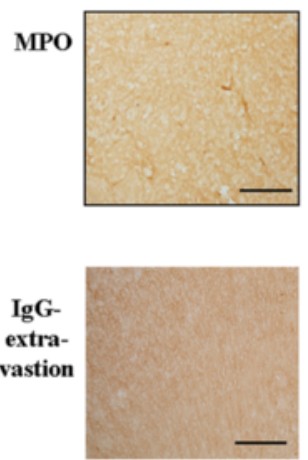

CXCL5

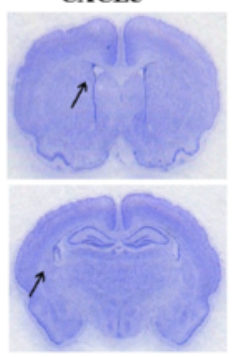

CXCL5

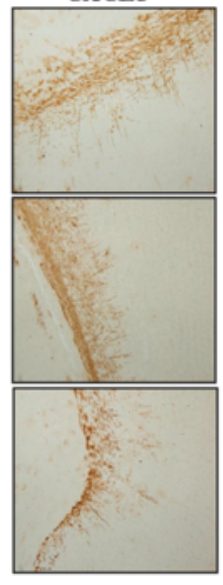

CXCL5

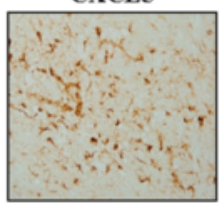

CXCL5
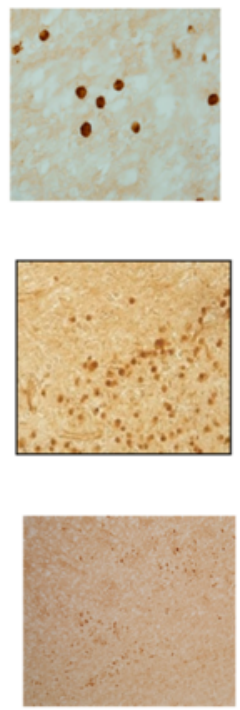

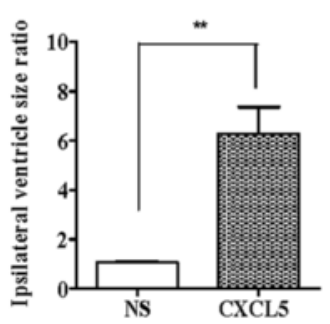

MBP
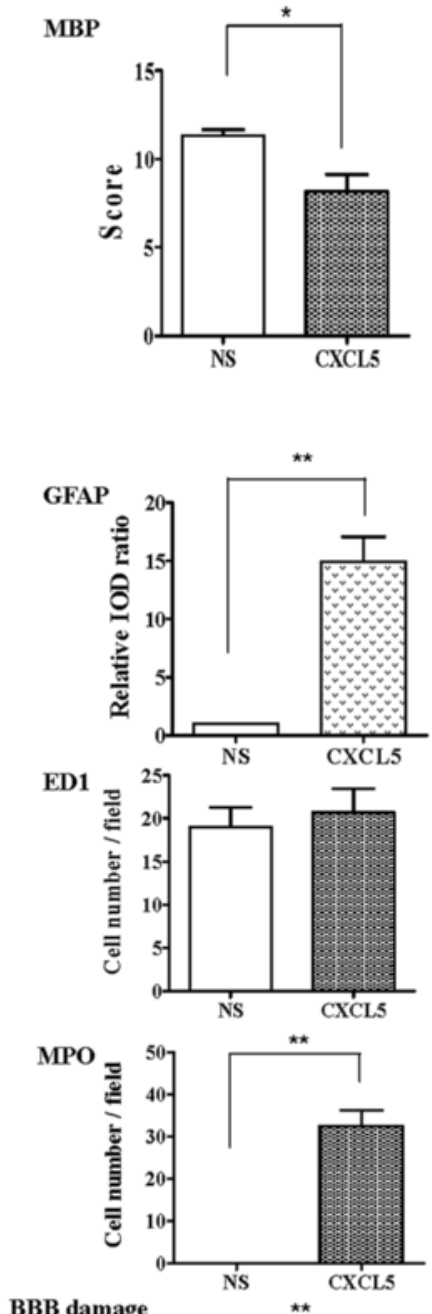

BBB damage

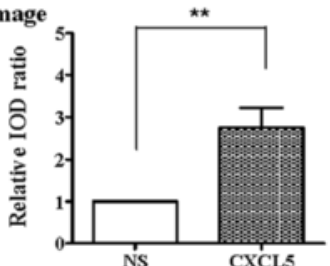

Fig. 7 (See legend on next page.) 
(See figure on previous page.)

Fig. 7 CXCL5 alone increased neutrophil infiltration and BBB damage and caused white matter injury. Intracerebroventricular infusion of recombinant CXCL5 $(2 \mu \mathrm{g})$ or NS on P2 revealed that the CXCL5 group $(n=6)$ had significantly higher ipsilateral ventricle size ratios $(\mathbf{a})$, reduced myelination (MBP) (b), and increased astrogliosis (GFAP) (c) in the white matter on P12 compared with the vehicle group $(n=6)$. Scale bar $=100 \mu \mathrm{m}$. (d) At $24 \mathrm{~h}$ after injection, the NS $(n=6)$ and CXCL5 groups $(n=6)$ showed no detectable ED1 $(+)$ activated microglia in the white matter. By contrast, the CXCL5 group had a significantly higher number of $\mathrm{MPO}(+)$ neutrophils and significantly greater BBB damage compared with the vehicle group. Scale bar $=50 \mu \mathrm{m}(\mathrm{ED} 1)$ and $100 \mu \mathrm{m}$ (IgG and MPO); values are means \pm SEMs, ${ }^{*} p<0.05,{ }^{* *} p<0.01$

[36]. Our previous studies showed that tumor necrosis factor-alpha (TNF- $\alpha$ )-mediated TNF receptor 1 signaling was an upstream pathway leading to JNK activation that mediated neuroinflammation and BBB damage and induced white matter injury in the immature brain $[18,23]$. CXCL5 can regulate the production of TNF- $\alpha$ and the activation of the NF-kB and MAPK pathways, including the JNK pathway [37]. Our study findings suggest that CXCL5 signaling may be an upstream pathway leading to JNK activation in the immature brain.

In addition to regulating inflammatory cell migration, chemokines are valuable in normal human brain development [38]. In this study, the CXCL5 expression in the neurons of the cerebral cortex of the P2 rat pups was higher than that of the P7 and P30 rats. The regional distribution of CXCL5 in the developing cerebral cortex may be related to the proliferation, migration, and differentiation of various cell types in the developing central nervous system [38]. All normal P2, P7, and P30 rats had extremely low CXCL5 expression in the white matter. By contrast, high CXCL5 expression was observed after selective white matter injury induced by LPS-sensitized $\mathrm{HI}$, indicating that CXCL5 may be a critical tissue injury response marker.

We and others have used IgG extravasation in the perfused brain as an indicator of BBB damage in the neonatal brain [18, 23, 25, 26]. All the animals were perfused before the brain were removed and processed for IgG staining. Extravascular IgG immunoreactivity in the brain after $\mathrm{HI}$ is located at cellular as well as parenchymal levels. IgG entry into the brain cells after ischemia has been described in studies using immunostaining [39-41]. Glia can rapidly take up plasma proteins from

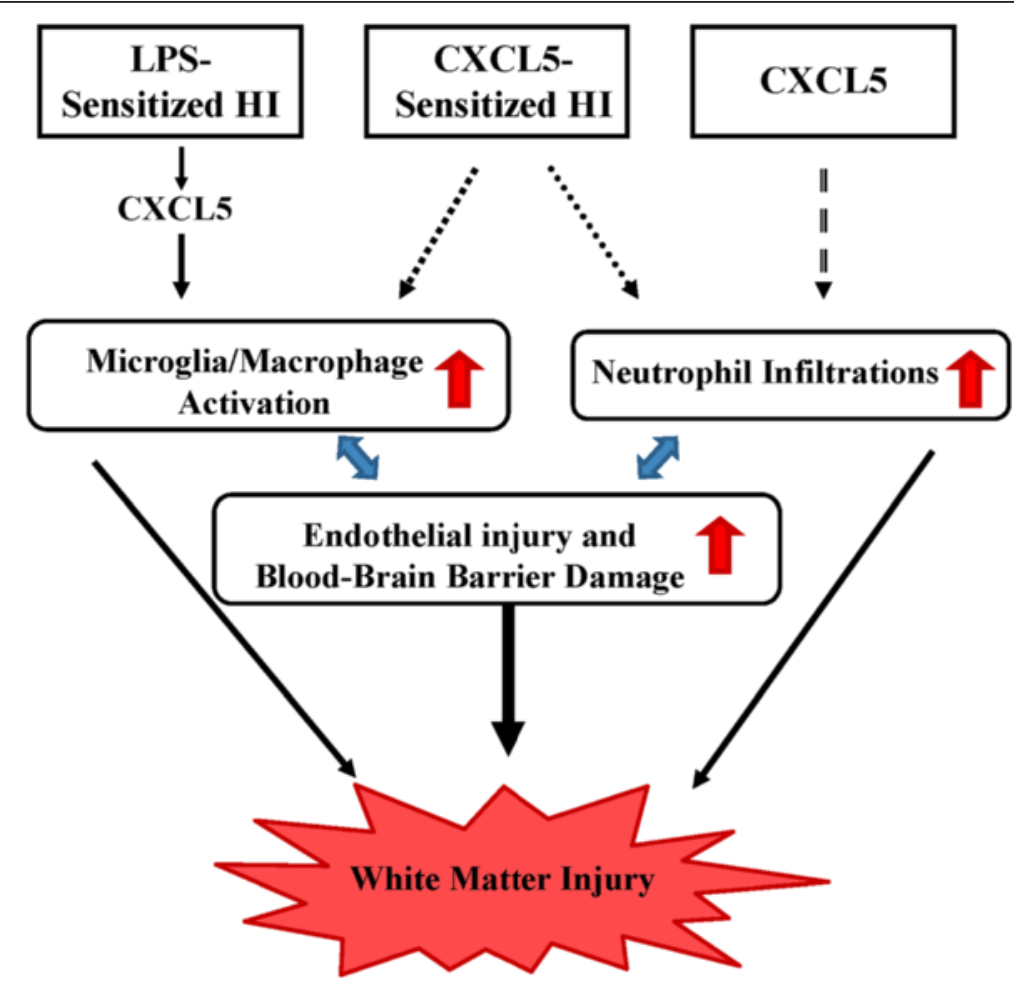

Fig. 8 Diagram showing that the proposed CXCL5-mediated signaling pathway, triggered by LPS-sensitized HI, CXCL5-sensitized HI, or CXCL5 alone, plays a crucial role in neuroinflammation and BBB disruption and subsequent white matter injury in the immature brain. Neuroinflammation and BBB damage are two mutually potentiating mechanisms leading to sustained neuroinflammation and BBB disruption in white matter injury of the developing brain. White matter injury can be induced by different neuroinflammatory mechanisms: predominant microglial activation by LPSsensitized $\mathrm{HI}$, microglial activation and neutrophil infiltration by CXCL5-sensitized $\mathrm{HI}$, and predominant neutrophil infiltration by CXCL5 alone 
the extracellular space of the injured brain through endocytosis [41]. Fc-receptors on reactive microglia may trap IgG in the tissue to facilitate its phagocytic activity [42]. In addition, extravasated plasma constituents after cerebral ischemia may act also as an inductive factor on microglial cells [43]. Additional sensitive markers for BBB damage are needed for more comprehensive assessment of BBB integrity in future studies of the neonatal brain injury.

\section{Conclusions}

White matter injury in preterm infants may not be associated with a single etiological pathway but may instead represent a generic tissue injury response of the white matter at a specific stage of development to various insults. Excessive recruitment of leukocytes is critical to the pathogenesis of white matter injury, and the magnitude and duration of the inflammatory process may ultimately determine the outcome of preterm infants with white matter injury. According to the results of our study, CXCL5 plays a central role as a converging point for upstream HI or infection and downstream neuroinflammation and BBB damage in the pathogenesis of white matter damage in the immature brain (Fig. 8). Our study findings suggest that CXCL5 may be a potential biomarker for white matter injury and cerebral palsy in preterm infants. Moreover, pharmacologically blocking CXCL5 signaling or its cognate receptor CXCR2 to attenuate dysregulated neuroinflammation is a potential strategy for treating white matter injury in the immature brain.

\section{Additional file}

Additional file 1: Supplementary methods. The measurement of cerebral ventricular size and quantification methods for gray matter injury and immunohistochemistry are described in detail here. (DOC 5563 kb)

\section{Abbreviations}

BBB: blood-brain barrier; GFAP: glial fibrillary acidic protein; HI: hypoxic ischemia; LPS: lipopolysaccharide; IgG: immunoglobulin G; IOD: integrated optical density; JNK: c-Jun N-terminal kinase; TNF-a: tumor necrosis factoralpha; MBP: myelin basic protein; NS: normal saline.

\section{Competing interests}

The authors declare that they have no competing interests.

\section{Authors' contributions}

LYW and CCH participated in the design and execution of the study, performed data analysis, and drafted the manuscript. YFT and YCL provided continual intellectual input and assisted with data interpretation. LYW and YFT made substantial technical support-related contributions to animal preparation. $\mathrm{CCH}$ conceived, designed, and coordinated the project and revised the manuscript critically for valuable intellectual content. All authors take public responsibility for the accuracy and integrity of the work and have read and approved the final manuscript.

\section{Acknowledgements}

This study was supported by grants from Chi Mei Medical Center (CMFHR9718), Taiwan Ministry of Science and Technology (MOST 103-2314-B-038-062-MY3), and the National Health Research Institutes (NHRI-EX101-9916NI). The sponsors had no roles in the design and conduct of the study; in the collection, analysis, and interpretation of the data; or in the preparation, review, and approval of the manuscript.

\section{Author details}

'Department of Pediatrics, Chi Mei Medical Center, Tainan 710, Taiwan. ${ }^{2}$ Department of Childhood Education and Nursery, Chia Nan University of Pharmacy and Science, Tainan, Taiwan. ${ }^{3}$ Department of Pediatrics, National Cheng Kung University Hospital, College of Medicine, National Cheng Kung University, Tainan 704, Taiwan. ${ }^{4}$ Institute of Clinical Medicine, College of Medicine, National Cheng Kung University, Tainan 704, Taiwan. ${ }^{5}$ Department of Pediatrics, College of Medicine, Taipei Medical University, Taipei 110, Taiwan. ${ }^{6}$ Department of Pediatrics, Wan-Fang Hospital, Taipei Medical University, Taipei 110, Taiwan.

Received: 16 August 2015 Accepted: 30 December 2015

Published online: 06 January 2016

\section{References}

1. Raines EW, Ferri N. Thematic review series: the immune system and atherogenesis. Cytokines affecting endothelial and smooth muscle cells in vascular disease. J Lipid Res. 2005;46:1081-92.

2. Charo IF, Ransohoff RM. The many roles of chemokines and chemokine receptors in inflammation. New Engl J Med. 2006;354:610-21.

3. Sepuru KM, Poluri KM, Rajarathnam K. Solution structure of CXCL5-a novel chemokine and adipokine implicated in inflammation and obesity. PLoS One. 2014:9:e93228.

4. Walz A, Schmutz P, Mueller C, Schnyder-Candrian S. Regulation and function of the CXC chemokine ENA-78 in monocytes and its role in disease. J Leukoc Biol. 1997:62:604-11.

5. Rovai LE, Herschman HR, Smith JB. The murine neutrophil-chemoattractant chemokines LIX, KC, and MIP-2 have distinct induction kinetics, tissue distributions, and tissue-specific sensitivities to glucocorticoid regulation in endotoxemia. J Leukoc Biol. 1998;64:494-502.

6. Chandrasekar B, Melby PC, Sarau HM, Raveendran M, Perla RP, Marelli-Berg FM, et al. Chemokine-cytokine cross-talk. The ELR+ CXC chemokine LIX (CXCL5) amplifies a proinflammatory cytokine response via a phosphatidylinositol 3kinase-NF-kappa B pathway. J Biol Chem. 2003;278:4675-86.

7. Volpe JJ. Brain injury in premature infants: a complex amalgam of destructive and developmental disturbances. Lancet Neurol. 2009:8:110-24.

8. Kadhim H, Tabarki B, Verellen G, De Prez C, Rona AM, Sebire G. Inflammatory cytokines in the pathogenesis of periventricular leukomalacia. Neurology. 2001;56:1278-84

9. Dammann O, Durums S, Leviton A. Do white cells matter in white matter damage? Trends Neurosci. 2001;24:320-4

10. Volpe JJ. Systemic inflammation, oligodendroglial maturation and encephalopathy of prematurity. Ann Neurol. 2011;70:525-9.

11. Keelan JA, Yang J, Romero RJ, Chaiworapongsa T, Marvin KW, Sato TA, et al. Epithelial cell-derived neutrophil-activating peptide-78 is present in fetal membranes and amniotic fluid at increased concentrations with intraamniotic infection and preterm delivery. Biol Reprod. 2004;70:253-9.

12. Deng $L Z$, Shi $C H$, Huang YM. Study on relations between the dynamic changes of serum HMGB1, ENA-78 levels and hypoxic-ischemic encephalopathy. Chin J Clinicians. 2010;4:1840-3.

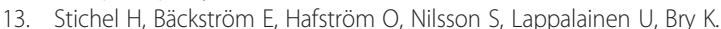
Inflammatory cytokines in gastric fluid at birth and the development of bronchopulmonary dysplasia. Acta Paediatr. 2011;100:1206-12.

14. MohanKumar K, Kaza N, Jagadeeswaran R, Garzon SA, Bansal A, Kurundkar $A R$, et al. Gut mucosal injury in neonates is marked by macrophage infiltration in contrast to pleomorphic infiltrates in adult: evidence from an animal model. Am J Physiol Gastrointest Liver Physiol. 2012;303:G93-102.

15. Back SA, Miller SP. Brain injury in premature neonates: a primary cerebral dysmaturation disorder? Ann Neurol. 2014;75:469-86.

16. Craig A, Luo NL, Beardsley DJ, Wingate-Pearse N, Walker DW, Hohimer AR, et al. Quantitative analysis of perinatal rodent oligodendrocyte lineage progression and its correlation with human. Exp Neurol. 2003;181:231-40.

17. Wang LW, Lin YC, Wang ST, Yeh TF, Huang CC. Hypoxic/ischemic and infectious events have cumulative effects on the risk of cerebral palsy in very-low-birth-weight preterm infants. Neonatology. 2014;106:209-15.

18. Wang LW, Tu YF, Huang CC, Ho CJ. JNK signaling is the shared pathway linking neuroinflammation, blood-brain barrier disruption, and 
oligodendroglial apoptosis in the white matter injury of the immature brain. J Neuroinflammation. 2012;9:175.

19. Viola A, Luster AD. Chemokines and their receptors: drug targets in immunity and inflammation. Annu Rev Pharmacol Toxicol. 2008;48:171-97.

20. Bento AF, Leite DF, Claudino RF, Hara DB, Leal PC, Calixto JB. The selective nonpeptide CXCR2 antagonist SB225002 ameliorates acute experimental colitis in mice. J Leukoc Biol. 2008;84:1213-21.

21. Lee HT, Chang YC, Tu YF, Huang CC. VEGF-ANEGFR-2 signaling leading to CREB phosphorylation is a shared pathway underlying the protective effect of preconditioning on neurons and endothelial cells. J Neurosci. 2009;29:4356-68.

22. Paxions $\mathrm{G}$, Watson $\mathrm{C}$. The rat brain in stereotaxic coordinates. 6th ed. Amsterdam: Elsevier; 2007.

23. Wang LW, Chang YC, Chen SJ, Tseng CH, Liao NS, Huang CC, et al. TNFR1-JNK signaling is the shared pathway of neuroinflammation and neurovascular damage after LPS-sensitized hypoxic-ischemic injury in the immature brain. J Neuroinflammation. 2014;11:215.

24. Hung PL, Huang CC, Tu DG, Huang HM, Chang YC. Thyroxin treatment protects against white matter injury in the immature brain via brain-derived neurotrophic factor. Stroke. 2013;44:2275-83.

25. Svedin P, Hagberg H, Savman K, Zhu C, Mallard C. Matrix metalloproteinase9 gene knock-out protects the immature brain after cerebral hypoxiaischemia. J Neurosci. 2007;27:1511-8.

26. Muramatsu K, Fukuda A, Togari H, Wada Y, Nishino H. Vulnerability to cerebral hypoxic-ischemic insult in neonatal but not in adult rats is in parallel with disruption of the blood-brain barrier. Stroke. 1997;28:2281-8.

27. Wang LW, Chang YC, Lin CY, Wang ST, Hong JS, Huang CC. Low-dose lipopolysaccharide selectively sensitizes hypoxic-ischemia-induced white matter injury in the immature brain. Pediatr Res. 2010;68:41-7.

28. Chew LJ, Takanohashi A, Bell M. Microglia and inflammation: impact on developmental brain injuries. Ment Retard Dev Disabil Res Rev. 2006;12: 105-12.

29. Hagberg H, Mallard C, Ferriero DM, Vannucci SJ, Levison SW, Vexler ZS, et al. The role of inflammation in perinatal brain injury. Nat Rev Neurol. 2015;11: 192-208.

30. Moretti R, Pansiot J, Bettati D, Strazielle N, Ghersi-Egea JF, Damante G, et al. Blood-brain barrier dysfunction in disorders of the developing brain. Front Neurosci. 2015;9:40.

31. Beck GC, Yard BA, Breedijk AJ, Van Ackern K, Van Der Woude FJ. Release of CXC-chemokines by human lung microvascular endothelial cells (LMVEC) compared with macrovascular umbilical vein endothelial cells. Clin Exp Immunol. 1999;118:298-303.

32. Koch AE, Kunkel SL, Harlow LA, Mazarakis DD, Haines GK. Epithelial neutrophil activating peptide-78: a novel chemotactic cytokine for neutrophils in arthritis. J Clin Invest. 1994;94:1012-8.

33. Shin JH, Park YM, Kim DH, Moon GJ, Bang OY, Ohn T, et al. Ischemic brain extract increases SDF-1 expression in astrocytes through the CXCR2/miR223/miR-27b pathway. Biochim Biophys Acta. 1839;2014:826-36.

34. Veenstra M, Ransohoff RM. Chemokine receptor CXCR2: physiology regulator and neuroinflammation controller? J Neuroimmunol. 2012;246:1-9.

35. Liu L, Darnall L, Hu T, Choi K, Lane TE, Ransohoff RM. Myelin repair is accelerated by inactivating CXCR2 on nonhematopoietic cells. J Neurosci. 2010;30:9074-83.

36. Lue H, Dewor M, Leng L, Bucala R, Bernhagen J. Activation of the JNK signalling pathway by macrophage migration inhibitory factor (MIF) and dependence on CXCR4 and CD74. Cell Signal. 2011:23:135-44.

37. Balamayooran G, Batra S, Cai S, Mei J, Worthen GS, Penn AL, et al. Role of CXCL5 in leukocyte recruitment to the lungs during secondhand smoke exposure. Am J Respir Cell Mol Biol. 2012;47:104-11.

38. Filipovic R, Jakovcevski I, Zecevic N. GRO-alpha and CXCR2 in the human fetal brain and multiple sclerosis lesions. Dev Neurosci. 2003;25:279-90.

39. Remmers M, Schmidt-Kastner R, Belayev L, Lin B, Busto R, Ginsberg MD. Protein extravasation and cellular uptake after high-dose human-albumin treatment of transient focal cerebral ischemia in rats. Brain Res. 1999; 827:237-42.

40. Simard JM, Geng Z, Woo SK, Ivanova S, Tosun C, Melnichenko L, et al. Glibenclamide reduces inflammation, vasogenic edema, and caspase-3 activation after subarachnoid hemorrhage. J Cereb Blood Flow Metab. 2009; 29:317-30.

41. Del Bigio MR, Deck JHN, Davidson GS. Glial swelling with eosinophilia in human post-mortem brains: a change indicative of plasma extravasation. Acta Neuropathol. 2000;100:688-94.
42. Jensen MB, Finsen B, Zimmer J. Morphological and immunophenotypic microglial changes in the denervated fascia dentata of adult rats: correlation with blood-brain barrier damage and astroglial reactions. Exp Neurol. 1997:143:103-16.

43. Jorgensen MB, Finsen BR, Jensen MB, Castellano B, Diemer NH, Zimmer J. Microglial and astroglial reactions to ischemic and kainic acid-induced lesions of the adult rat hippocampus. Exp Neurol. 1993;120:70-88.

\section{Submit your next manuscript to BioMed Central and we will help you at every step:}

- We accept pre-submission inquiries

- Our selector tool helps you to find the most relevant journal

- We provide round the clock customer support

- Convenient online submission

- Thorough peer review

- Inclusion in PubMed and all major indexing services

- Maximum visibility for your research

Submit your manuscript at www.biomedcentral.com/submit
Biomed Central 